Please do not remove this page

RMIT

UNIVERSITY

\title{
Surface gap solitons at fabricated photonic lattice interfaces
}

Rosberg, Christian; Neshev, Dragomir; Krolikowski, Wieslaw; Mitchell, Arnan; Vicencio, Rodrigo; Molina, Mario; Kivshar, Yuri

https://researchrepository.rmit.edu.au/esploro/outputs/9921862771401341/filesAndLinks?institution=61RMIT_INST\&index=null

Rosberg, C., Neshev, D., Krolikowski, W., Mitchell, A., Vicencio, R., Molina, M., \& Kivshar, Y. (2006). Surface gap solitons at fabricated photonic lattice interfaces. Proceedings of the 31st Australian Conference on Optical Fibre Technology and Australian Optical Society (ACOFT\&AOS 2006), 130-132.

https://doi.org/10.1109/ACOFT.2006.4519298

Published Version: https://doi.org/10.1109/ACOFT.2006.4519298

Repository homepage: https://researchrepository.rmit.edu.au

(C2006 IEEE. Personal use of this material is permitted. However, permission to reprint/republish this material for advertising or promotional purposes or for creating new collective works for resale or redistribution to servers or lists, or to reuse any copyrighted component of this work in other works must be obtained from the IEEE.

Downloaded On 2023/04/26 22:14:39 +1000 


\title{
Surface gap solitons at fabricated photonic lattice interfaces
}

\author{
Christian R. Rosberg ${ }^{1,2}$, Dragomir N. Neshev ${ }^{1}$, Wieslaw Krolikowski ${ }^{2}$, Arnan Mitchell ${ }^{3}$, \\ Rodrigo A. Vicencio ${ }^{4}$, Mario I. Molina ${ }^{5}$, and Yuri S. Kivshar ${ }^{1}$ \\ ${ }^{1}$ Nonlinear Physics Centre and ${ }^{2}$ Laser Physics Centre, \\ Centre for Ultrahigh-bandwidth Devices for Optical Systems (CUDOS), \\ Research School of Physical Sciences and Engineering, \\ Australian National University, Canberra, Australia \\ E-mail: crr124@rsphysse.anu.edu.au \\ ${ }^{3}$ School of Electrical and Computer Systems Engineering, RMIT University, Melbourne, Australia \\ ${ }^{4}$ Max-Planck-Institut für Physik komplexer Systeme, Dresden, Germany \\ ${ }^{5}$ Departamento de Física, Facultad de Ciencias, Universidad de Chile, Santiago
}

\begin{abstract}
We generate surface gap solitons with staggered phase structure at the edge of a semi-infinite $\mathrm{LiNbO}_{3}$ waveguide array with defocusing nonlinearity. We characterize selflocalization dynamics and identify the threshold power for soliton formation.
\end{abstract}

\section{INTRODUCTION}

Surface waves in periodic systems were first introduced in 1932 by Tamm [1], who considered a truncated electronic potential which under certain conditions was found to support localized states at the boundary (surface). Such states are now commonly referred to as "Tamm states". Because of the difficulties in observing this type of surface waves in natural materials such as crystals, successful efforts have been made to experimentally demonstrate their existence at the interfaces of nano-engineered periodic layered structures or superlattices [2]. In optics, linear surface waves have been observed in periodic GaAs-AlGaAs structures [3], exploiting the well-known analogy between wave physics in optical and electronic periodic systems.

In this work, we demonstrate experimentally the existence of self-localized optical surface waves at the edge of a defocusing waveguide array acting as a semi-infinite nonlinear photonic lattice. We observe, for the first time to our knowledge, the formation of surface gap solitons, recently predicted theoretically [4].

Surface gap solitons are self-localized surface modes with a staggered phase structure and a propagation constant lying within the first photonic transmission bandgap [5]. The defocusing nonlinear response of the material enables localization in the form of surface modes with alternating phase structure inside the periodic structure, thus allowing for a full nonlinear analogy with the electronic Tamm states.

Nonlinear surface waves with trivial phase structure, trapped entirely due to the mechanism of total internal reflection were recently observed at the edge of self-focusing waveguide arrays [6]. They differ substantially from the surface gap states, for which the localization mechanism consists of total internal reflection from the homogeneous region combined with Bragg reflection from the periodic structure.
The observation of optical surface modes in practical fabricated photonic structures opens an avenue for technical applications in areas such as dynamic micro-sensing and alloptical switching and beam steering in integrated information processing systems.

\section{EXPERIMENTAL SETUP}

The experiment is performed in a semi-infinite array of closely spaced single mode waveguides, fabricated by Titanium indiffusion in a mono-crystal lithium niobate wafer. The $\mathrm{LiNbO}_{3}$ sample exhibits a strong photovoltaic effect which leads to a self-defocusing nonlinearity.

In the fabrication process, $100 \AA$ of $\mathrm{Ti}$ was deposited on the $\mathrm{X}$-cut $\mathrm{LiNbO}_{3}$ using electron beam evaporation. The Ti layer was then photolithographically patterned and etched in a buffered hydrofluoric acid solution. The diffusion was conducted at $1050^{\circ} \mathrm{C}$ for 3 hours in a wet oxygen environment. The waveguides were verified to be single mode using a prism coupling technique. The array was diced to a total length of $50 \mathrm{~mm}$ and both facets were mechanically polished. The refractive index contrast is $\Delta \mathrm{n}=3 \cdot 10^{-4}$, the waveguide spacing $\mathrm{d}=9.0 \mu \mathrm{m}$, and the number of waveguides 100. Inset in Fig. 1(b) schematically illustrates the photonic lattice geometry.

At the input face of the sample, a circular shaped and extraordinarily polarized probe beam from a cw Nd: $\mathrm{YVO}_{4}$ laser at wavelength $\lambda=532 \mathrm{~nm}$ is focused by a microscope objective $(\times 20)$ to a full width at half-maximum $(\mathrm{FWHM})$ of $3 \mu \mathrm{m}$, and injected into the waveguide at the edge of the array. Provided the refractive index contrast exceeds a certain threshold [7], such single-site excitation can efficiently generate nonlinear localized modes with staggered phase structure in this type of defocusing waveguide arrays. In this case the periodic structure appears fully equivalent to a discrete system [7].

The propagated wave packet at the sample output is imaged onto a CCD camera to capture the intensity and phase distributions. 

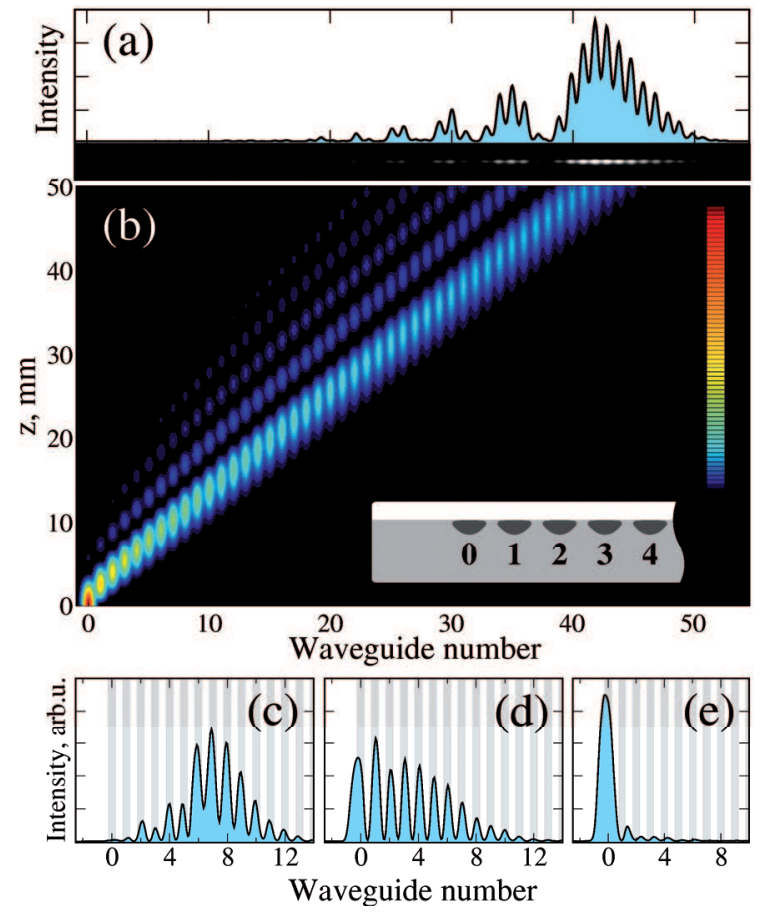

Figure 1. (a) Measured and (b) calculated linear discrete diffraction of a narrow low power beam $(0.1 \mu \mathrm{W})$ when only the edge waveguide is excited. Inset in (b) illustrates the waveguide geometry. (c-e) Surface gap soliton formation in the nonlinear regime. Pictures show the output intensity distribution at times 920,1050 , and $1550 \mathrm{~s}$, respectively, after beam power is increased to $0.50 \mathrm{~mW}$. Grey shading marks the waveguide positions.

\section{LINEAR SURRFACE REPULSION AND DIFFRACTION}

At low laser power $(0.1 \mu \mathrm{W})$ the probe beam experiences discrete diffraction and spreads out in the horizontal plane upon propagation, due to the strong coupling between neighboring waveguides. Fig. 1(a) depicts the experimental output image and the corresponding transverse intensity profile in this situation. After linear propagation through the array the beam profile spans more than ten waveguides, and is centered approximately 42 lattice sites away from the input excitation point, indicating strong surface repulsion. Fig. 1(b) shows the corresponding theoretical intensity distribution inside the sample, calculated using an analytical formula [8]. The excellent agreement between the experimental observation and the theoretical prediction yields a value of $0.46 \mathrm{~mm}^{-1}$ for the intersite coupling coefficient of the waveguide array, implying a total longitudinal propagation of 23 coupling lengths.

\section{NONLINEAR SURFACE SELF-TRAPPING}

Increasing the laser power leads to spatial beam self-action in the nonlinear regime. The slow response of the photovoltaic nonlinearity allows us to monitor the dynamics of the soliton formation, providing additional information about the localization process. Figures 1(c-e) show the output beam intensity profile at times 920, 1050, and $1550 \mathrm{~s}$, respectively, after the beam power is increased to $0.50 \mathrm{~mW}$. The wave packet is seen to first contract and move towards

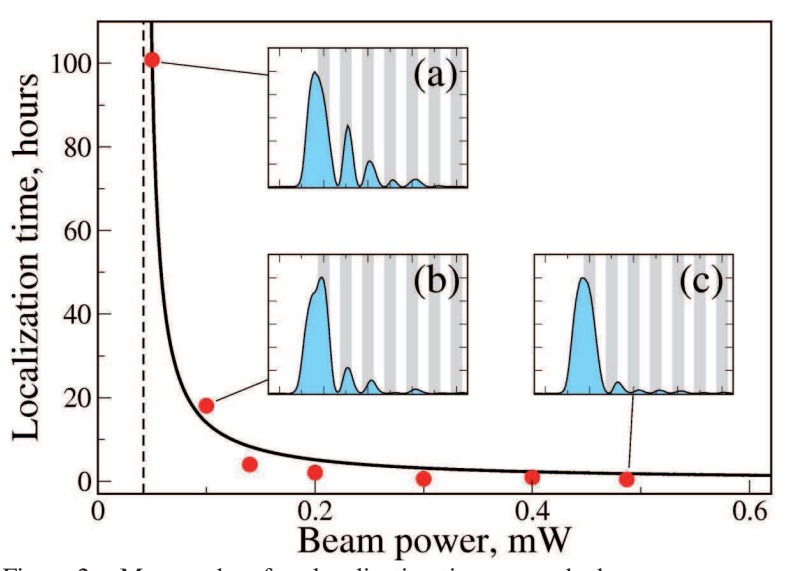

Figure 2. Measured surface localization time vs. probe beam power. Solid curve: $A+B /\left(P-P_{t h}\right)$ fit to experimental data (red dots). Vertical dashed line marks the threshold power $\left(\mathrm{P}_{\mathrm{th}}=0.042 \mathrm{~mW}\right)$. (a-c) Beam intensity profiles of decreasing width corresponding to the indicated points.

the edge of the array [Fig. 1(c)]. Then, in Fig. 1(d), partial self-trapping in the surface waveguide with a tail of intensity lobes extending into the periodic medium becomes apparent. A series of zero intensity points between these lobes indicates the self-induced dynamic formation of a staggered phase structure. Eventually, a strongly localized surface gap soliton is formed [Fig. 1(e)].

The defocusing nonlinearity effectively decreases the contrast of the surface waveguide, causing the mode to broaden and penetrate into the homogeneous region. The asymmetry of the photonic structure is reflected in the shape of the trapped beam which monotonically decays above the surface, but shows damped oscillations inside the periodic region.

\section{THRESHOLD CHARACTERISTICS}

In the low-power linear regime the probe beam was seen to diffract and be repelled from the lattice surface [Figs. 1(a, b)].

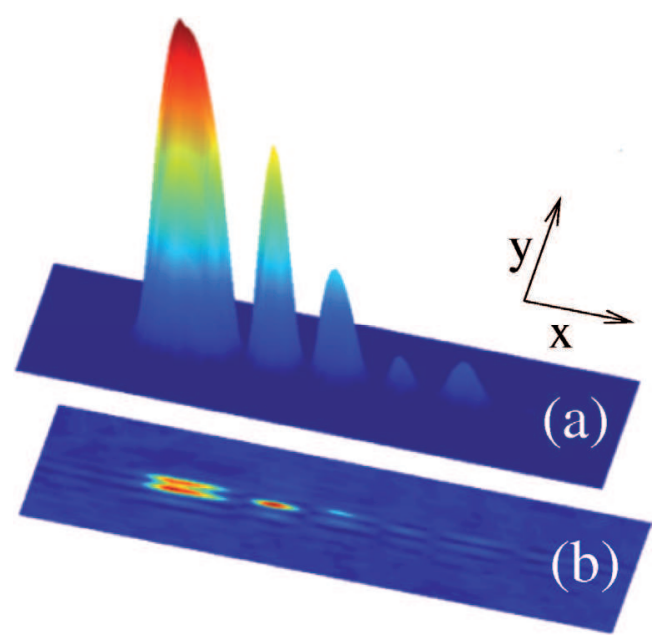

Figure 3. (a) Three-dimensional representation of the surface gap soliton observed experimentally near the threshold power [corresponding to Fig. 2(a)], (x,y) are the transverse coordinates. (b) Plane-wave interferogram demonstrating the staggered phase structure of the surface gap soliton. 
In stark contrast to this, increasing the laser power by two orders of magnitude induced a strong nonlinear response and the formation of a localized surface state [Fig. 1(e)].

In order to study in detail the crossover between these very different regimes, we measure the formation time of the surface gap soliton as a function of the probe beam power. The results are summarized in Fig. 2. The formation time increases dramatically for decreasing input power until, below a certain critical power, no localized surface mode is observed. The observed critical slowing down indicates the existence of a threshold power below which the nonlinear response is too weak to cause self-trapping. The value of the threshold power was estimated as $\mathrm{P}_{\text {th }}=0.042 \mathrm{~mW}$ by modeling the dynamics of the soliton formation time, fitting the function $\mathrm{A}+\mathrm{B} /\left(\mathrm{P}-\mathrm{P}_{\mathrm{th}}\right)$ [Fig. 2, solid curve] to the experimental data [Fig. 2, red dots].

Figures 2 (a-c) show the beam intensity profiles corresponding to the indicated data points. The width of the localized mode decreases for increasing beam power, spanning about three lattice sites immediately above threshold [see Fig. 2(a)], and approximately a single lattice site at higher power, as indicated in Fig. 2(c). Decreasing beam width is observed due to the fact that stronger beam selfaction at higher power leads to a deeper surface defect, and hence more pronounced beam localization.

\section{GAP SOLITON PHASE STRUCTURE}

An essential and unique feature of surface gap solitons is the staggered phase structure of the beam tail inside the periodic medium. The alternating phase of the field lobes reflects the fact that the propagation constant of the selflocalized mode lies within the Bragg reflection gap of the transmission spectrum. To verify this nontrivial phase structure in the experiment we interfere the output beam with a vertically inclined broad plane reference beam and image the obtained interferogram [Fig. 3]. Fig. 3(a) depicts a three dimensional representation of the spatial beam intensity distribution of a broad surface gap soliton observed near the threshold power $(0.05 \mathrm{~mW})$. Fig. 3(b) shows an intensity plot of the associated interference pattern, where $\mathrm{x}$ and $\mathrm{y}$ are the transverse coordinates. A half-period vertical (y) shift of the interference fringes, corresponding to an exact $\pi$ phase jump in the horizontal beam direction (x), is clearly observed between each pair of lobes in the structure [Fig. 3(b)].

\section{NONLINEAR DISCRETE MODEL}

To get a deeper insight into the physics of the surface gap soliton formation and the transition from linear surface repulsion to nonlinear self-trapping, we consider a nonlinear discrete model consisting of a system of coupled-mode equations for the normalized waveguide mode amplitudes.

While no localized surface modes exist in the linear regime, as this would require much larger index contrast between the waveguides and the continuum, the presence of defocusing nonlinearity can give rise to localized states in the model. To find them we solve numerically the corresponding
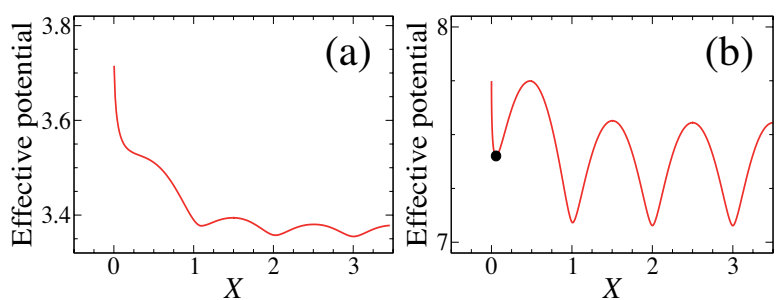

Figure 4. (a,b) Effective potential vs. the collective coordinate $X$ below and above the threshold power, respectively. Integer values of $X$ correspond to the waveguide numbers. The black dot in (b) marks the stationary localized surface state that appears above threshold.

stationary equations by a multi-dimensional Newton-Raphson scheme.

A detailed analysis of the stationary states and their transformations by a numerical constraint method allows us to obtain the effective energy that describes both the mode interaction with the surface and the trapping by the discrete lattice as a function of the transverse collective coordinate $X$. Stable localized states, corresponding to the minima of this function, are found to appear only above a certain power threshold, as clearly observed in the experiment (Section V). Figures $4(a, b)$ show the effective potential for two cases above and below the threshold power, respectively.

\section{CONCLUSIONS}

We have demonstrated in experiment that gap solitons can be generated near the surface of a periodic medium with selfdefocusing nonlinearity in the form of staggered surface modes, providing the first evidence of a nonlinear analog of localized surface Tamm states in optics. The dynamic nature of the nonlinearity-controlled wave propagation demonstrated in this work shows promising perspectives for all-optical switching and beam steering applications.

\section{ACKNOWLEDGEMENT}

The authors thank A.A. Sukhorukov for useful discussions. This work was supported by the Australian Research Council.

\section{REFERENCES}

[1] I. E. Tamm, "A possible kind of electron binding on crystal surfaces," Z. Phys. 76, 849 (1932).

[2] H. Ohno et al., "Observation of "Tamm states" in superlattices,” Phys. Rev. Lett. 64, 2555-2558 (1990).

[3] P. Yeh, A. Yariv, and A. Y. Cho, "Optical surface waves in periodic layered media,” Appl. Phys. Lett. 32, 104-105 (1978).

[4] Y. V. Kartashov, V. A. Vysloukh, and L. Torner, ' Surface gap solitons," Phys. Rev. Lett. 96, 073901 (2006).

[5] Yu. S. Kivshar and G. P. Agrawal, "Optical Solitons: From Fibers to Photonic Crystals", Academic Press, San Diego, 2003.

[6] S. Suntsov et al., "Observation of discrete surface solitons," Phys. Rev. Lett. 96, 063901 (2006).

[7] M. Matuszewski et al., "Crossover from self-defocusing to discrete trapping in nonlinear waveguide arrays," Optics Express 14, 254-259 (2006).

[8] K. G. Makris et al., "Discrete surface solitons," Opt. Lett. 30, 2466-2468 (2005). 